\title{
THE RESTRICTED VARIATIONAL BAYES APPROXIMATION IN BAYESIAN FILTERING
}

\author{
Václav Šmídl \\ Academy of Sciences, Prague, Czech Republic
}

Anthony Quinn

\author{
Trinity College Dublin, Ireland
}

\begin{abstract}
The Variational Bayes (VB) approach is used as a one-step approximation for Bayesian filtering. It requires the availability of moments of the free-form distributional optimizers. The latter may have intractable functional forms. In this contribution, we replace these by appropriate fixed-form distributions yielding the required moments. We address two scenarios of this Restricted VB (RVB) approximation. For the first scenario, an application in identification of HMMs is given. In the second, the fixed-form distribution is generated via Particle Filtering (PF). It is shown that a new approximation of Rao-Blackwellized particle filtering is obtained in this scenario of RVB. Its performance is assessed for a simple nonlinear model.
\end{abstract}

\section{THE VB APPROXIMATION}

The VB approximation is a deterministic free-form optimization technique. It was first used in off-line inference problems [1] and extended to on-line inference of time-invariant parameters in [2]. The use of VB in Bayesian filtering was first discussed in [3]. The key theory is now reviewed.

Theorem 1 (Variational Bayes (VB)) Let $f(\theta \mid D)$ be the posterior distribution of multivariate parameter, $\theta=\left[\theta_{1}^{\prime}, \theta_{2}^{\prime}\right]^{\prime}$, and $\breve{f}(\theta \mid D)$ be an approximate distribution restricted to the set of conditionally independent distributions

$$
\breve{f}(\theta \mid D)=\breve{f}\left(\theta_{1}, \theta_{2} \mid D\right)=\breve{f}\left(\theta_{1} \mid D\right) \breve{f}\left(\theta_{2} \mid D\right) .
$$

The minimum of the Kullback-Leibler divergence

$$
\tilde{f}(\theta \mid D)=\arg \min _{\breve{f}(\cdot)} K L(\breve{f}(\theta \mid D) \| f(\theta \mid D)),
$$

is reached for

$$
\tilde{f}\left(\theta_{i} \mid D\right) \propto \exp \left(\mathrm{E}_{\tilde{f}\left(\theta_{/ i} \mid D\right)}[\ln (f(\theta, D))]\right), \quad i=1,2,
$$

where $\theta_{/ i}$ denotes the complement of $\theta_{i}$ in $\theta$. We will refer to $\tilde{f}(\theta \mid D)$ (7) as the VB-approximation, and $\tilde{f}\left(\theta_{i} \mid D\right)$ (3) as the VB-marginals.

The above theorem provides a powerful tool for approximation of joint pdfs in separable form [3]:

$$
\ln f\left(\theta_{1}, \theta_{2}, D\right)=g\left(\theta_{1}, D\right)^{\prime} h\left(\theta_{2}, D\right) .
$$

Here, $g\left(\theta_{1}, D\right)$ and $h\left(\theta_{2}, D\right)$ are finite-dimensional vectors of compatible dimension. Using (4) in (3),

$$
\tilde{f}\left(\theta_{1} \mid D\right) \propto \exp \left(g\left(\theta_{1}, D\right)^{\prime} h\left(\widehat{\theta_{2}, D}\right)\right),
$$

where $\widehat{h(\cdot)}=\mathrm{E}_{\tilde{f}\left(\theta_{2} \mid D\right)}[h(\cdot)]$ are the VB-moments for $\theta_{2}$, and similarly for $\theta_{1}$. An Iterative VB (IVB) [3] moment-swapping algorithm is implied. In many non-linear cases of $g$ and/or $h$, the VB-marginals (3) are non-standard in form, and so the required VB-moments are difficult to evaluate. In this contribution, we aim to replace any such non-standard VB-marginal with a tractable alternative, as follows.

Corollary 1 (of Theorem 1: Restricted VB) Let $\breve{f}(\theta \mid D)$ be a conditionally-independent approximation of $f(\theta \mid D)$ of the kind

$$
\breve{f}(\theta \mid D)=\breve{f}\left(\theta_{1}, \theta_{2} \mid D\right)=\breve{f}\left(\theta_{1} \mid D\right) \bar{f}\left(\theta_{2} \mid D\right),
$$

where $\bar{f}\left(\theta_{2} \mid D\right)$ is a fixed-form distribution. Then, the minimal KL divergence (2), under (6), is reached for

$$
\tilde{f}\left(\theta_{1} \mid D\right) \propto \exp \left(\mathrm{E}_{\bar{f}\left(\theta_{2} \mid D\right)}[\ln (f(\theta, D))]\right) .
$$

$\bar{f}\left(\theta_{2} \mid D\right)$ needs to be chosen judiciously, such that its momentsrequired in (7) — are available. These moments are substituted just once, without the need for IVB cycles. Some standard distributional approximation methods may be interpreted as special cases of RVB; e.g. (i) certainty equivalence, where $\bar{f} \equiv \delta\left(\theta_{2}-\hat{\theta}_{2}\right)$, in which case (7) becomes the conditional, $f\left(\theta_{1} \mid D, \hat{\theta}_{2}\right)$; and (ii) the Quasi-Bayes $(Q B)$ approximation, where $\bar{f} \equiv f\left(\theta_{2} \mid D\right)$, the exact marginal, if available [3].

\section{BAYESIAN FILTERING}

Consider the following model structure

$$
d_{t} \sim f\left(d_{t} \mid \theta_{t}\right), \quad \theta_{t} \sim f\left(\theta_{t} \mid \theta_{t-1}\right)
$$

where $\theta_{t}$ is known as the state variable. By Bayesian Filtering $(\mathrm{BF})$, we mean the recursive evaluation of the filtering distribution $f\left(\theta_{t} \mid D_{t}\right)$ using Bayes' rule. $D_{t}=\left[d_{1}, \ldots, d_{t}\right]$ denotes the history of observations. The computational flowchart of BF is displayed in Fig. 1. BF is analytically tractable if (i) 


$$
\begin{aligned}
& f\left(\theta_{t} \mid \theta_{t-1}, D_{t-1}\right) \quad f\left(d_{t} \mid \theta_{t}, D_{t-1}\right) \text { Scenario I }
\end{aligned}
$$

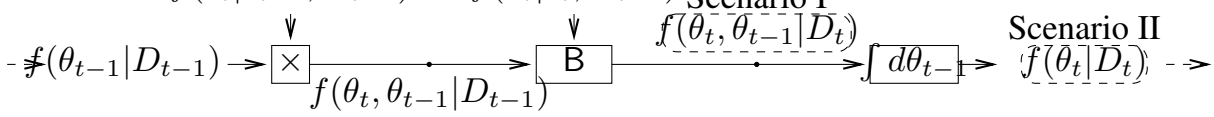

Fig. 1. Two possible points ('scenarios') for VB approximation are indicated in a computational flowchart of exact Bayesian filtering. $\times$ denotes multiplication, $\mathrm{B}$ a Bayes' rule step, and $\int d \cdot$ marginalization over the indicated variable.

marginalization over $\theta_{t-1}$ is analytically tractable, and (ii) the resulting marginal distribution, $f\left(\theta_{t} \mid D_{t}\right)$, is in the same form as the previous step, $f\left(\theta_{t-1} \mid D_{t-1}\right)$, so that the procedure can be iterated. We call this condition conjugacy in the BF context. (i) and (ii) are satisfied only for a very limited class of models.

\subsection{Particle Filtering}

Particle filtering $(P F)[4]$ refers to a range of techniques for generating an empirical approximation of $f\left(\Theta_{t} \mid D_{t}\right)$ via importance sampling:

$$
\tilde{f}\left(\Theta_{t} \mid D_{t}\right)=\sum_{i=1}^{n} w_{t}^{(i)} \delta\left(\Theta_{t}-\Theta_{t}^{(i)}\right), w_{t}^{(i)} \propto \frac{f\left(\Theta_{t}^{(i)} \mid D_{t}\right)}{q\left(\Theta_{t}^{(i)} \mid D_{t}\right)}
$$

Here, $\Theta_{t}=\left[\theta_{1}, \ldots, \theta_{t}\right]$ is the state trajectory, $\Theta_{t}^{(i)}, i=$ $1, \ldots, n$ are random samples drawn from an appropriately chosen importance function, $q\left(\Theta_{t} \mid D_{t}\right)$, and $w_{t}^{(i)}$ are the importance weights.

In the Rao-Blackwellized $\mathrm{PF}$ (RBwPF), the state variable is partitioned as $\theta_{t}=\left[\theta_{1, t}, \theta_{2, t}\right]$. An empirical approximation of the marginal is then sought:

$$
\begin{aligned}
\tilde{f}\left(\Theta_{2, t} \mid D_{t}\right) & =\sum_{i=1}^{n} w_{t}^{(i)} \delta\left(\Theta_{2, t}-\Theta_{2, t}^{(i)}\right) \\
w_{t}^{(i)} & \propto \frac{f\left(d_{t} \mid \theta_{2, t}^{(i)}\right) f\left(\theta_{2, t}^{(i)} \mid \theta_{2, t-1}^{(i)}\right)}{q\left(\theta_{2, t}^{(i)} \mid D_{t}, \Theta_{2, t-1}^{(i)}\right)} w_{t-1}^{(i)} .
\end{aligned}
$$

The numerator of (11) requires integration over $\theta_{1, t-1}$. Hence, $\mathrm{RBwPF}$ is feasible if there exists a partition of the state variable allowing this integration to be performed analytically. From (10), the implied approximation of the other marginal is

$$
\tilde{f}\left(\theta_{1, t} \mid D_{t}\right)=\sum_{i=1}^{n} w_{t}^{(i)} f\left(\theta_{1, t} \mid D_{t}, \Theta_{2, t}^{(i)}\right) .
$$

\section{VARIATIONAL BAYESIAN FILTERING (VBF)}

VB provides the possibility for deterministic approximation of the filtering distribution [3]. We now examine two scenarios for its application, as indicated in Fig. 1.

Scenario I of VBF: If marginalization over $\theta_{t-1}$ (Fig. 1) is intractable, it can be replaced by VB-marginalization. This is achieved by forcing conditional independence between $\theta_{t}$ and $\theta_{t-1}$ in $f\left(\theta_{t}, \theta_{t-1} \mid D_{t}\right)$ (Fig 1). The joint distribution needed in (3) is:

$$
\begin{aligned}
& f\left(d_{t}, \theta_{t}, \theta_{t-1} \mid D_{t-1}\right)= \\
& \quad f\left(d_{t} \mid \theta_{t}, D_{t-1}\right) f\left(\theta_{t} \mid \theta_{t-1}\right) \tilde{f}\left(\theta_{t-1} \mid D_{t-1}\right) .
\end{aligned}
$$

Application of Theorem 1 to (13) yields VB-marginals in the form of two parallel Bayes' rule updates:

$$
\begin{aligned}
\tilde{f}\left(\theta_{t} \mid D_{t}\right) & \propto f\left(d_{t} \mid \theta_{t}, D_{t-1}\right) \tilde{f}\left(\theta_{t} \mid D_{t-1}\right), \\
\tilde{f}\left(\theta_{t-1} \mid D_{t}\right) & \propto \tilde{f}\left(d_{t} \mid \theta_{t-1}, D_{t-1}\right) \tilde{f}\left(\theta_{t-1} \mid D_{t-1}\right) .
\end{aligned}
$$

The following approximate distributions are involved:

$$
\begin{gathered}
\tilde{f}\left(\theta_{t} \mid D_{t-1}\right) \propto \exp \left\{\mathrm{E}_{\tilde{f}\left(\theta_{t-1} \mid D_{t}\right)}\left[\ln f\left(\theta_{t} \mid \theta_{t-1}\right)\right]\right\}, \\
\tilde{f}\left(d_{t} \mid \theta_{t-1}, D_{t-1}\right) \propto \exp \left\{\mathrm{E}_{\tilde{f}\left(\theta_{t} \mid D_{t}\right)}\left[\ln f\left(\theta_{t} \mid \theta_{t-1}\right)\right]\right\} .
\end{gathered}
$$

From (16), the form of $\tilde{f}\left(\theta_{t} \mid D_{t-1}\right)$ is determined by the form of the time-invariant parameter evolution model. Hence, one application of Bayes' rule (14) yields the same functional form at each time, satisfying the condition of conjugacy [3].

Scenario II of VBF: Consider the case where marginalization over $\theta_{t-1}$ is tractable (Fig 1), but yields a filtering distribution, $f\left(\theta_{t} \mid D_{t}\right)$, in a form different from that at $t-1$, violating conjugacy. However, if the VB approximation is then used to force conditional independence with respect to the partition $\theta_{t}=\left[\theta_{1, t}, \theta_{2, t}\right]$, these VB-marginals may be invariant, restoring conjugacy.

\section{RESTRICTED VB IN BAYESIAN FILTERING}

We now employ the RVB approximation (Corollary 1) for the two scenarios of VBF outlined in the previous section. Note, however, that there are many choices for the fixed distribution, $\bar{f}(\cdot)$, and that the final decision will depend on the particular model structure (8).

\subsection{Scenario I}

The moments of $\tilde{f}\left(\theta_{t-1} \mid D_{t}\right)$ (15) required in (16) may be intractable. An obvious fixed-form replacement of this distribution is the VB-filtering distribution from the previous step:

$$
\bar{f}\left(\theta_{t-1} \mid D_{t}\right) \equiv \tilde{f}\left(\theta_{t-1} \mid D_{t-1}\right) .
$$

The VB-filtering distribution is then obtained without iterations, by substituting moments of $\tilde{f}\left(\theta_{t-1} \mid D_{t-1}\right)$ via (16), and the result into (14). 


\subsection{Scenario II: RVB+PF}

For the first time, we propose a stochastic approximation as the fixed-form distribution (Corollary 1):

$$
\bar{f}\left(\theta_{2, t} \mid D_{t}\right)=\sum_{i=1}^{n} \bar{w}_{t}^{(i)} \delta\left(\theta_{2, t}-\theta_{2, t}^{(i)}\right) .
$$

Following the standard RBwBF approach of Section 2.1, the particle weights are approximated as

$$
\begin{gathered}
\bar{w}_{t}^{(i)} \propto \tilde{f}\left(d_{t} \mid \theta_{2, t}^{(i)}\right) f\left(\theta_{2, t}^{(i)} \mid \theta_{2, t-1}^{(i)}\right) \bar{w}_{t-1}^{(i)} / q\left(\theta_{2, t}^{(i)} \mid D_{t}, \Theta_{2, t-1}^{(i)}\right) . \\
\tilde{f}\left(d_{t} \mid \theta_{2, t}\right)=\int f\left(d_{t} \mid \theta_{t}\right) \tilde{f}\left(\theta_{1, t} \mid D_{t-1}\right) d \theta_{1, t} .
\end{gathered}
$$

which is obtained from (9) using (i) the assumption of independent observation models for $\theta_{1, t}$ and $\theta_{2, t}$, and (ii) VBmarginals $\tilde{f}\left(\theta_{t-1} \mid D_{t-1}\right)$ in place of exact marginals $f\left(\theta_{t-1} \mid D_{t-1}\right)$.

Hence, the VB-marginal, $\tilde{f}\left(\theta_{1, t} \mid D_{t}\right)$, must be tractable. From (4) and (19), it is given by

$$
\tilde{f}\left(\theta_{1, t} \mid D_{t}\right) \propto \exp \left(g\left(\theta_{1, t}\right)^{\prime} \sum_{i=1}^{n} \bar{w}_{t}^{(i)} h\left(\theta_{2, t}^{(i)}, D_{t}\right)\right) .
$$

In the sequel, we will call this the $R V B+P F$ approximation.

Remark 1 The computational flow of $R B w P F$ and $R V B+P F$ differ in the following respects: (i) $R B w P F$ requires analytical marginalization of the whole trajectory $\Theta_{1, t-1}$ (i.e. conjugacy at each step (Section 2)), while RVB+PF is tractable at every step if the one-step marginalization in (21) is tractable (i.e. conjugacy is not required); and (ii) one set of sufficient statistics is stored for each particle, $\Theta_{2, t}^{(i)}$, in RBwPF (12), while one set of sufficient statistics is used for all the samples $\theta_{2, t}^{(i)}$ in $R V B+P F(22)$.

Remark 2 The proposed $R V B+P F$ scheme generalizes the Modified RBwPF method (MRBwPF) proposed in [5], where $f\left(\theta_{1, t} \mid D_{t}\right)$ was approximated by certainty equivalence; i.e.

$$
\tilde{f}\left(\theta_{1, t} \mid D_{t}\right) \propto \exp \left(g\left(\theta_{1, t}\right)^{\prime} h\left(\sum_{i=1}^{n} \bar{w}_{t}^{(i)} \theta_{2, t}^{(i)}, D_{t}\right)\right),
$$

and $f\left(d_{t} \mid D_{t-1}, \theta_{2, t}\right)$ in (20) was approximated using sampling. In the simulations which follow, we will use MRBwPF with (20) for easier comparison. Results using the samplingbased approximation for $w_{t}^{(i)}$ [5] are very similar.

\section{SIMULATION STUDIES}

\subsection{Inference of HMM with Unknown Transition Matrix}

Consider a HMM with the following two constituents: (i) a first-order Markov chain on the unobserved discrete (label) variable $l_{t}$, with $c$ possible states and time-variant unknown transition matrix $T_{t} \in \Re^{c \times c}$; and (ii) a set of $c$ known

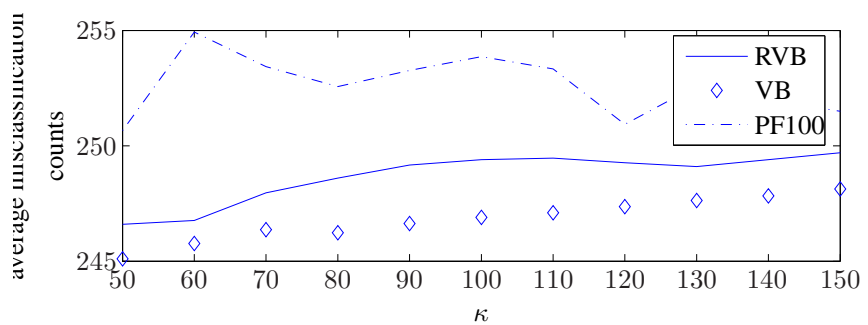

Fig. 2. Performance of competing algorithms for rapidlyvarying $T_{t}\left(\kappa_{\text {true }}=80\right)$.

class-conditional observation models, as arises in classification. The problem of inferring $l_{t}$ can be formalized as a task of Bayesian filtering as follows:

$$
\begin{array}{r}
l_{t} \sim \mathcal{M} u_{l_{t}}\left(T_{t} l_{t-1}\right), \quad T_{t} \sim \mathcal{D} i_{T_{t}}\left(\kappa T_{t-1}+\mathbf{1}_{c, c}\right), \\
d_{t} \sim f_{1}\left(d_{t}\right)^{l_{1, t}} \times \ldots \times f_{c}\left(d_{t}\right)^{l_{c, t}} .
\end{array}
$$

Here, scalar parameter $\kappa$ governs variance of $T_{t}$. For high values of $\kappa$, the variance of the Dirichlet distribution is very tight allowing only slow evolution of $T_{t}$, while low values of $\kappa$ allow faster evolution of $T_{t}$.

Under Scenario I (Section 3), the VB-approximation yields:

$$
\begin{aligned}
\tilde{f}\left(l_{t} \mid D_{t}\right) & =\mathcal{M} u_{l_{t}}\left(\alpha_{t}\right), \tilde{f}\left(T_{t} \mid D_{t}\right)=\mathcal{D} i_{T_{t}}\left(Q_{t}\right), \\
\tilde{f}\left(l_{t-1} \mid D_{t}\right) & =\mathcal{M} u_{l_{t}}\left(\beta_{t}\right), \tilde{f}\left(T_{t-1} \mid D_{t}\right) \propto \exp \left(q\left(T_{t-1}\right)\right),
\end{aligned}
$$

where shaping parameters $\alpha_{t}, Q_{t}, \beta_{t}$ are omitted for brevity. $q\left(T_{t-1}\right)$ is a complicated function, and so the moments of $\tilde{f}\left(T_{t-1} \mid D_{t}\right)$ are analytically intractable. Therefore, we make the RVB assignment (18),

$$
\bar{f}\left(T_{t-1} \mid D_{t}\right) \equiv \tilde{f}\left(T_{t-1} \mid D_{t-1}\right),
$$

which is Dirichlet (24).

A Monte Carlo study (100 runs) was undertaken, in order to compare (i) this RVB approach with (ii) (full) VB (i.e. (24), with numerical evaluation of $\tilde{f}\left(T_{t-1} \mid D_{t}\right)$ on a grid of $100 \times 100$ points), and with (iii) a particle filter with 100 particles (PF100) (Figure 2). Performance was defined in terms of the average number of misclassified labels in 1000 data samples. The RVB scheme is computationally cheaper than the competing methods, yet offers good performance even for rapid variations of $T_{t}$ (Figure 2). For slowly varying $T_{t}$ (high $\kappa)$, the RVB performance is comparable to VB.

\subsection{Performance of RVB+PF (Scenario II)}

Consider the following model:

$$
\begin{aligned}
f\left(x_{t} \mid x_{t-1}\right) & =\mathcal{N}\left(A x_{t-1}, Q\right), \\
f\left(C_{t} \mid C_{t-1}\right) & =\mathcal{N}\left(\arctan \left(C_{t-1}\right), P\right), \\
f\left(d_{t} \mid x_{t}, C_{t}\right) & =\mathcal{N}\left(C_{t}^{\prime} x_{t}, R\right) .
\end{aligned}
$$


Essentially, this is a standard linear-Gaussian model with unknown non-stationary $C_{t}$, for which a non-linear evolution model is defined. Here, integration over $x_{t-1}$ is possible using standard Kalman Filtering (KF) theory, yielding the following conditional posterior of $x_{t}$ :

$$
\begin{gathered}
f\left(x_{t} \mid D_{t}, C_{t}\right)=\mathcal{N}_{x_{t} \mid C_{t}}\left(\mu_{t}, \Omega_{t}^{-1}\right), \\
\Omega_{t}=\left(Q+A \Omega_{t-1}^{-1} A^{\prime}\right)^{-1}+C_{t}^{\prime} R^{-1} C_{t}, \\
\mu_{t}=\Omega_{t}^{-1}\left[\left(Q+A \Omega_{t-1}^{-1} A^{\prime}\right)^{-1} A \mu_{t-1}+C_{t}^{\prime} R^{-1} d_{t}\right] .
\end{gathered}
$$

This is written in terms of precision matrix $\Omega_{t}$ for analytical convenience. Exact integration over $C_{t-1}$ is intractable. A Rao-Blackwellized PF is obtained using (10)-(11) with assignments $\theta_{1, t}=x_{t}$ and $\theta_{2, t}=C_{t}$.

Since the log of (25) can be written as $g\left(x_{t}\right) h\left(C_{t}, D_{t}\right)$ (4), it is amenable to RVB+PF approximation (Section 4.2). The resulting VB-marginal, $\tilde{f}\left(x_{t} \mid D_{t}\right)$ is Gaussian, i.e. in the form of (25), with assignments

$$
\begin{aligned}
\Omega_{t} & =\left(Q+A \Omega_{t-1}^{-1} A^{\prime}\right)^{-1}+\mathrm{E}\left[C_{t}^{\prime} R^{-1} C_{t}\right] \\
\mu_{t} & =\Omega_{t}^{-1}\left[\left(Q+A \Omega_{t-1}^{-1} A^{\prime}\right)^{-1} A \mu_{t-1}+\widehat{C}_{t}^{\prime} R^{-1} d_{t}\right]
\end{aligned}
$$

where $\widehat{C_{t}}=\sum_{i=1}^{n} w_{t}^{(i)} C_{t}^{(i)}$. (26) is again in standard $\mathrm{KF}$ form, except for the term

$$
\mathrm{E}\left[C_{t}^{\prime} R^{-1} C_{t}\right]=\sum_{i=1}^{n} w_{t}^{(i)} C_{t}^{(i){ }^{\prime}} R^{-1} C_{t}^{(i)} .
$$

Thus the matrix inversion lemma cannot be easily applied and the implementation is less efficient than for standard KF.

MRBwPF (Remark 2) also yields a result in the form of (26), with (27) replaced by ${\widehat{C_{t}}}^{\prime} R^{-1} \widehat{C_{t}}$. This has the following consequences: (i) MRBwPF can use KF without any modification, while implementation of RVB+PF typically requires modification of KF; (ii) MRBwPF uses a certainty-equivalent estimate of $C_{t}$, while RVB+PF uses the second non-central moment (27), encoding information about its uncertainty.

A scalar system with one-dimensional state and output was simulated with parameters $A=1, Q=0.5, P=0.1$, $R=0.5$. The aim is to illustrate the effect of propagation of higher moments (27) within the KF scheme. Hence, we use exactly the same particle filter on $C_{t}$-i.e. the same importance function and re-sampling scheme-for all tested methods. The performance was assessed via the value of the posterior distribution of $X_{t}$ at the simulated value. Variants of $\tilde{f}\left(x_{t} \mid D_{t}\right)$-obtained from (25) via, respectively, RBwPF (12), RVB+PF (22), and MRBwPF (23) - are displayed in Table 1 . These numbers are obtained by averaging over 100 simulation runs for each tested setting. Similar behaviour was observed for other noise levels and numbers of particles.

Both RVB+PF and MRBwPF perform significantly worse than RBwPF but at much lower computational cost, since they require only one step of $\mathrm{KF}$ per time-step, in contrast to $n$

\begin{tabular}{c|ccc|c} 
number of data & RBwPF & RVB+PF & MRBPF & scale \\
\hline 100 & -0.37 & -2.58 & -2.68 & $\times 10^{3}$ \\
1000 & -0.58 & -3.43 & -3.44 & $\times 10^{5}$
\end{tabular}

Table 1. Results of MC studies displayed via log of the posterior distribution of $X_{t}$, evaluated at the simulated value (the greater the mantissa, the better the result).

Kalman updates for RBwPF. RVB+PF slightly outperforms MRBwPF but at the price of evaluation of the second-order moment (27) and the numerical overhead of incorporating this into the KF (26).

\section{CONCLUSION}

The VB approximation in Bayesian filtering is constrained by the need to evaluate moments of the resulting VB-marginals, which is difficult to ensure. If at least one of the VB-marginals is tractable, the method can be combined with other approximations via the Restricted VB (RVB) approach. In this paper, we have presented two possible Scenarios for the use of VB in Bayesian filtering, and for each Scenario one possible RVB scheme to overcome the intractability of VB-marginals. The first Scenario enforced conditional independence between the current and one-step-delayed state variable, and it is expected to work well for extensions of discrete hidden Markov models. The second Scenario enforced conditional independence in the posterior distribution at each time, $t$. In this Scenario, particle filtering was used to generate the fixed-form distribution. This yielded a new variant of Rao-Blackwellized particle filters, generalizing previous heuristically-motivated approximations. The new RVB-based particle filter is expected to offer a computationally far cheaper scheme than standard Rao-Blackwellized particle filters.

\section{REFERENCES}

[1] C. M. Bishop, "Variational principal components," in Proceedings of the Ninth International Conference on Artificial Neural Networks, (ICANN), 1999.

[2] M. Sato, "Online model selection based on the variational Bayes," Neural Computation, vol. 13, pp. 1649-1681, 2001.

[3] V. Šmídl and A. Quinn, The Variational Bayes Method in Signal Processing. Springer, 2005.

[4] A. Doucet, N. de Freitas, and N. Gordon, eds., Sequential Monte Carlo Methods in Practice. Springer, 2001.

[5] F. Mustière, M. Bolić, and M. Bouchard, "A modified Rao-Blackwellised particle filter," in Proceedings of the IEEE conference on Acoustics, Speech, and Signal Processing, 2006. 